\section{Investigating the Effect of Air Cavities of Sinuses on the Radiotherapy Dose Distribution Using Monte Carlo Method}

\author{
Seif F. ${ }^{1}$, Bayatiani M. R. ${ }^{1 *}$, Hamidi S. ${ }^{2}$, Kargaran M. ${ }^{3}$
}

\begin{abstract}
Background: Considering that some vital organs exist in the head and neck region, the treatment of tumors in this area is a crucial task. The existence of air cavities, namely sinuses, disrupt the radiotherapy dose distribution. The study aims to analyze the effect of maxillary, frontal, ethmoid and sphenoid sinuses on radiotherapy dose distribution by Monte Carlo method.
\end{abstract}

Material and Methods: In order to analyze the effect of the cavities on dose distribution, the maxillary, frontal, ethmoid and sphenoid sinus cavities were simulated with $(3 \times 3.2 \times 2) \mathrm{cm}^{3},(2 \times 2 \times 3.2) \mathrm{cm}^{3},(1 \times 1 \times 1.2) \mathrm{cm}^{3}$ and $(1 \times 1 \times 2) \mathrm{cm}^{3}$ dimensions.

Results: In the analysis of the dose distribution caused by cavities, some parameters were observed, including: inhomogeneity of dose distribution in the cavities, inhomogeneity of dose on the edges of the air cavities and dispersion of the radiations after the air cavity. The amount of the dose in various situations showed differences: before the cavity a $0.64 \%$ and a $2.76 \%$ decrease, a $12.06 \%$ and a $17.17 \%$ decrease in the air zone, and a $2.25 \%$ and a $5.9 \%$ increase after the cavity.

Conclusion: The results indicate that a drop in dose before the air cavities and in the air zone occurs due to the lack of scattered radiation. Furthermore, the rise in dose was due to the passage of more radiation from the air cavity and dose deposition after the air cavity. The changes in dose distribution are dependent on the cavity size and depth. As a result, this has to be noted in the treatment planning and MU calculations of the patient.

\section{Keywords}

Air Cavities, Monte Carlo Method, Radiotherapy Dose Distribution

\section{Introduction}

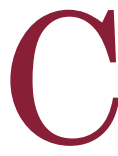

onsidering the existence of vital organs in the head and neck region, the proper treatment of the tumors located in this area in a way that critical organs would not be damaged is crucial. A tumor might take shape in the proximity of air cavities such as ethmoid, maxillary, frontal and sphenoid sinuses [1]. The existence of these air cavities creates changes in the radiotherapy dose distribution, and consequently, in the treatment calculations in the area. In regard to the evergrowing number of cancer patients and the importance of the treatment in the head and neck region, the effect of the air cavities in dose distribution was contributed to the optimum treatment planning and better care of Organs at Risks (OAR) close to the tumor. Over the past years, the assessment of the effect of air inhomogeneities on absorbed dose has been the subject of research in many empirical studies using the Monte Carlo simulation. In fact, the Monte Carlo simulation is one of the most
${ }^{1}$ Ph.D of Medical Phys-

ics. Assistant professor,

Department of Medical

Physics and Radio-

therapy, Arak university

of Medical Sciences and

Khansari hospital, Arak,

Iran

${ }^{2} \mathrm{Ph}$.D of Physics. Associ-

ate professor, Depart-

ment of Physics, Arak

University, Arak, Iran

${ }^{3}$ Ms.c of Physics, Depart-

ment of Physics, Arak

University, Arak, Iran

*Corresponding author:

M. R. Bayatiani

Ph.D of Medical Physics, Arak University of Medical Sciences \& Radiation Oncology Department of Khansari Hospital, Arak, Iran

E-mail: bayatiani@arakmu.ac.ir

Received: 5 November 2018 Accepted: 9 December 2018 
accurate calculation methods for radiotherapy dose $[2,3]$. In the studies carried out in this field, the occurrence of disorders due to the air inhomogeneities close to the intersection of air-tissue, and consequently, overdose and underdose in the surrounding areas have been reported [4-10]. For instance, when treating a tumor close to an air cavity in the head and neck region, underdose could occur in the intersection of air-tissue $[11,12]$. In this case, the possibility of the cancer recurrence close to the intersection of air-tissue increases [1]. In a study, Behrens et al. analyzed the effect of dose build up/down in the presence of air cavities. The results indicated that the effect of dose build up/down depends on the size of the field and the energy of the radiated beams and it also is generally more frequently seen for fields with smaller sizes and higher energies [13]. Apparently, the amount of change in dose in the intersection of air-tissue depends on the geometry and the size of the cavity; in other words, this depends on the electronic inequivalence [11, 14-17].

However, changes in the radiation dose have not yet been properly known owing to the existence of air cavities in the treatment volume, especially in the intersection of air-tissue (when the tumor is in its proximity) [15]. The purpose of this study is to use the Monte Carlo simulation for calculating and analyzing radiotherapy dose distribution in the presence of air cavities of ethmoid, maxillary, frontal and sphenoid sinuses.

\section{Material and Methods}

In this study, the head of the Varian 2100C/D accelerator was simulated using the factory information in the energy of $6 \mathrm{MV}$ via the MCNPX (Monte Carlo N-Particle eXtended) code. The simulated parts of the head in the accelerator were: source, target (two layers made of tungsten and copper), primary collimator (made of tungsten), vacuum window (two layers comprised of vacuum and beryllium), flattening filter (pyramid-shaped with
20 layers made of copper), ionization chamber (seven layers made from Kapton), mirror (having a 35 degree angle with the horizon and made from Mylar) and two pairs of collimators of $y$ and $x$ made from tungsten. Figure 1 depicts the main parts of the head of the linear accelerator which is simulated using the MCNPX code.

Gaussian distribution function was used to define the electron source. The cut-off energies for electron and photon were assumed to be 0.511 and $0.01 \mathrm{Mev}$, respectively. The input file was executed for 24 hours by a supercomputer at Isfahan University, which had a processor with $4 \times 64 G B$ nodes. In order to validate the simulation, the curves of depth dose percentage were calculated using the Monte Carlo method in $S S D=100 \mathrm{~cm}$ and for the reference field of $10 \times 10 \mathrm{~cm}^{2}$ and dose profile in the same $S S D$ and field, at depth of $5 \mathrm{~cm}$ in a $50 \times 50 \times 50 \mathrm{~cm}^{3}$ water phantom and the results were compared with the data obtained from dosimetry. To calculate the PDD in simulation, a cylinder with $0.5 \mathrm{~cm}$ radius and $30 \mathrm{~cm}$ height, along the $\mathrm{z}$ axis and on the central axis of the beam inside the phantom was simulated in a program. Moreover, to calculate dose profile, a similar cylinder perpendicular to the central axis of the beam along the $\mathrm{x}$ axis and at depth of $5 \mathrm{~cm}$ from the surface of the phantom was simulated in another program.

Experimental dosimetry was conducted in a

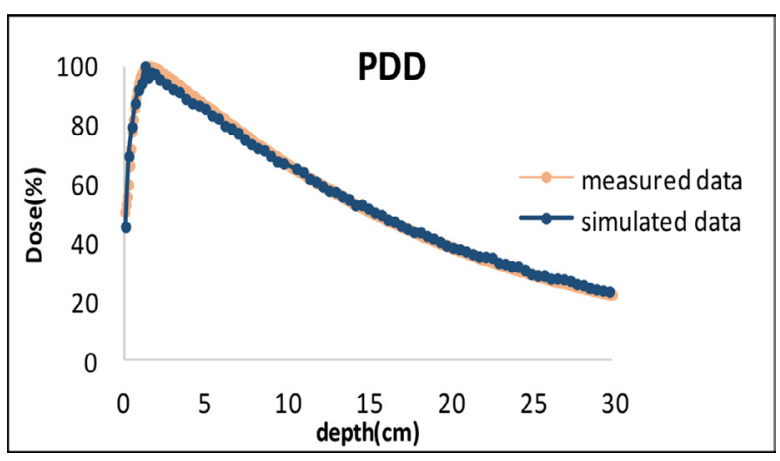

Figure 1: PDD curves calculated by Monte Carlo simulation and measured by the Ionizing chamber, field size $10 \times 10 \mathrm{~cm}^{2}$. 
3D water phantom, with the brand of "Blue

Water Phantom" and dimensions of $50 \times 50 \times 50$ $\mathrm{cm}^{3}$, manufactured by Scanditronix- Wellhöferm, using cylindrical ionizing chambers $\left(C C 13,0.13 \mathrm{~cm}^{3}\right)$.

In the next phase, four cube-shaped air cavities of sinuses with dimensions close to reality in certain depths of water phantom were programed as follows: $3 \times 3.2 \times 2 \mathrm{~cm}^{3}$ maxillary sinus at depth of $3.2 \mathrm{~cm}, 2 \times 2 \times 3.2 \mathrm{~cm}^{3}$ frontal sinus at the depth of $1.6 \mathrm{~cm}, 1 \times 1 \times 1.2 \mathrm{~cm}^{3}$ ethmoid sinus at depth of $2 \mathrm{~cm}$, and $1 \times 1 \times 2 \mathrm{~cm}^{3}$ sphenoid sinus at depth of $6 \mathrm{~cm}$ form phantom surface. When the cavities existed, PDD and Profile curves were drawn. In order to calculate the difference of dose distribution, these curves were compared in the presence and absence of air cavities.

\section{Results}

Validation of the carried out simulation

agreement with experimental dosimetry (PDD less than 2\% and Profile less than 4\%) Figures 1 and 2 respectively.

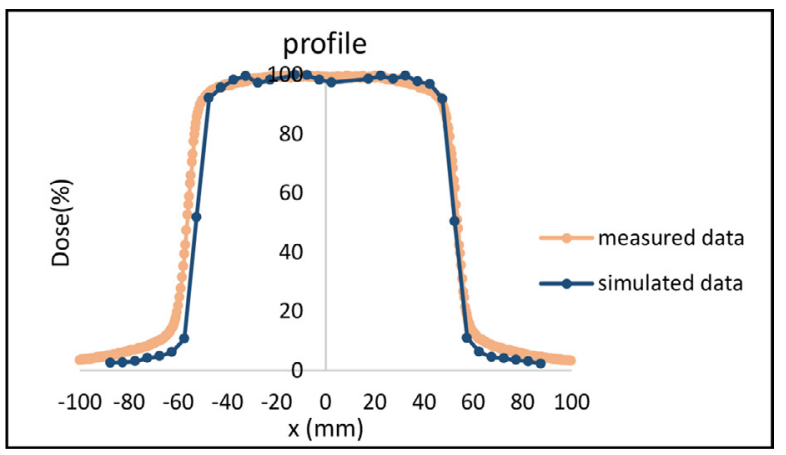

Figure 2: Profile curves calculated by Monte Carlo simulation and measured by the lonizing chamber, field size $10 \times 10 \mathrm{~cm}^{2}$.

\section{Investigating the impact of sinus- es on dose distribution Maxillary sinus}

Figure 3 shows changes in PDD in the presence and absence of the maxillary sinus. When there is a cavity, the depth of maximum dose

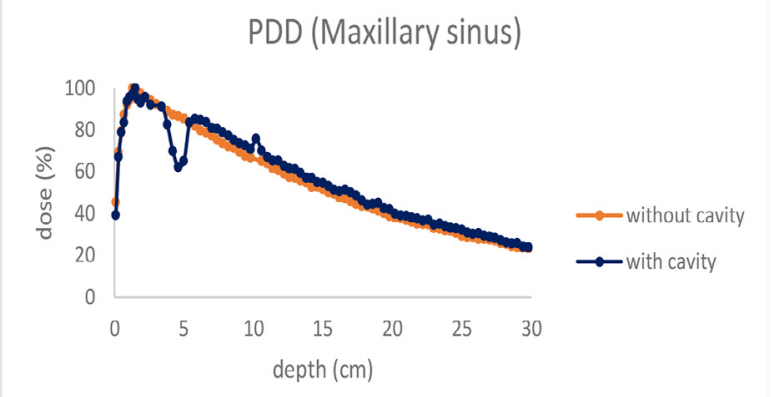

Figure 3: Comparing PDD curves in the presence and absence of maxillary sinus.

$\left(d_{m}\right)$ has shifted from $1.4 \mathrm{~cm}$ to $1.6 \mathrm{~cm}$. The air cavity leads to fluctuations in the dose distribution curve such that we observe a reduction of $1.45 \%$ in dose in the area before the maxillary sinus, a $17.17 \%$ dose reduction inside the air area, and a $3.95 \%$ increase in dose after the sinus.

Figure 4 indicates the values of dose profile in the phantom in the presence and absence of maxillary sinus ( $\mathrm{x}$-axis, $\mathrm{d}=5 \mathrm{~cm}$ ). When the sinus is in the transverse area of $-1.25 \mathrm{~cm}$ to $1.25 \mathrm{~cm}$, dose reduction takes place, since the maxillary sinus is located at the depth of 3.2 $\mathrm{cm}$ to $5.2 \mathrm{~cm}$ in this area. The dose reduction is $19.24 \%$.

The margins of error for PDD and profile calculations were $\pm 1.85 \% \mathrm{~cm}$ and $\pm 3.5 \% \mathrm{~cm}$, respectively.

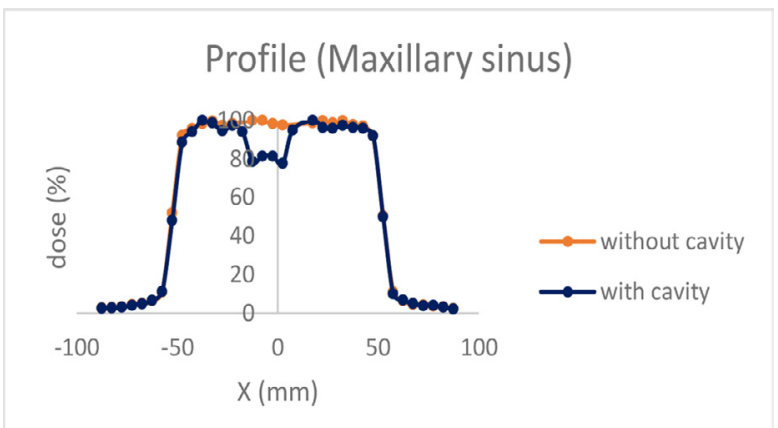

Figure 4: Comparing profile curves in the presence and absence of maxillary sinus. 


\section{Frontal sinus}

Figure 5 shows the comparison between values in the presence and absence of frontal sinus. In this case, the maximum spot of the dose $\left(d_{m}\right)$ shifts from $1.4 \mathrm{~cm}$ to $1.6 \mathrm{~cm}$. We see a $2.01 \%$ dose reduction before frontal sinus, a $15.9 \%$ dose reduction in the air area, a 5.9\% increase in dose after sinus, and discontinuity at the edge of the air cavity.

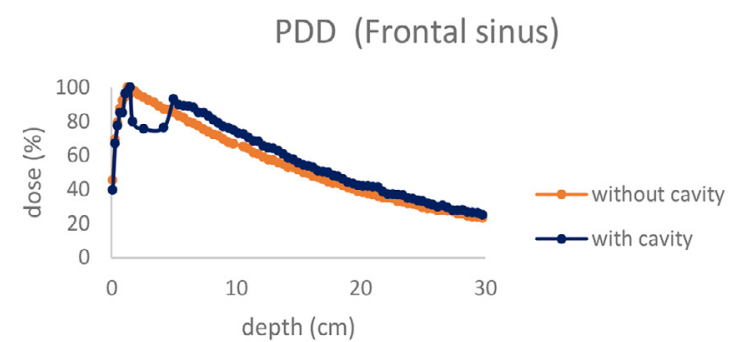

Figure 5: Comparing PDD curves in the presence and absence of frontal sinus.

Figure 6 indicates dose profile values $(d=$ $5 \mathrm{~cm})$ in the presence and absence of frontal sinus. In the transversal movement from -1.25 $\mathrm{cm}$ to $1.25 \mathrm{~cm}$, a small increase in dose (approximately $1.24 \%$ ) occurs since the frontal sinus is located at depth of $1.6 \mathrm{~cm}$ to $4.8 \mathrm{~cm}$, that it means it is located before dose profile cylinder. We also see that dose increase after the air cavity.

The margins of error for PDD and profile calculations were $\pm 2.27 \%$ and $\pm 2.4 \%$, respectively.

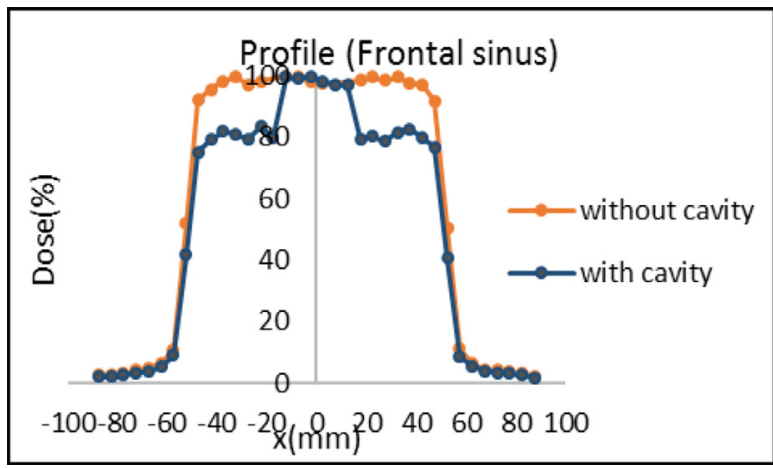

Figure 6: Comparing profile curves in the presence and absence of frontal sinus.
Ethmoid sinus

Figure 7 is a comparison between PDD curves in the presence and absence of ethmoid sinus. The maximum spot of the dose (1.4 $\mathrm{cm}$ ) has remained unchanged. We observed a $0.64 \%$ dose reduction before the ethmoid sinus, a $12.06 \%$ dose reduction in the air area, and a $2.3 \%$ dose increase after air cavity.

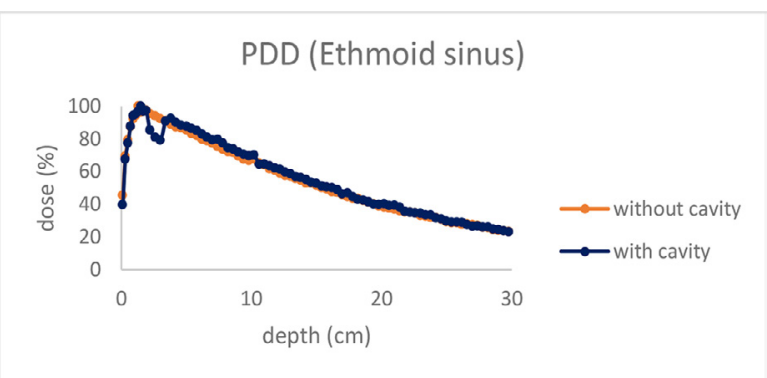

Figure 7: Comparing PDD curves in the presence and absence of ethmoid sinus.

Figure 8 shows a comparison between dose profile values $(d=5 \mathrm{~cm})$ in the presence and absence of ethmoid sinus. As is seen, the presence of ethmoid does not impose significant changes on the transversal dose. Only in the $-0.75 \mathrm{~cm}$ to $0.75 \mathrm{~cm}$ area, we can see a small amount of increase, since dimensions of the cavity are small and dose profile value after the sinus cavity has been calculated. Ethmoid is located at depth of $2 \mathrm{~cm}$ to $3.2 \mathrm{~cm}$ from the phantom surface.

The margins of error for PDD and profile

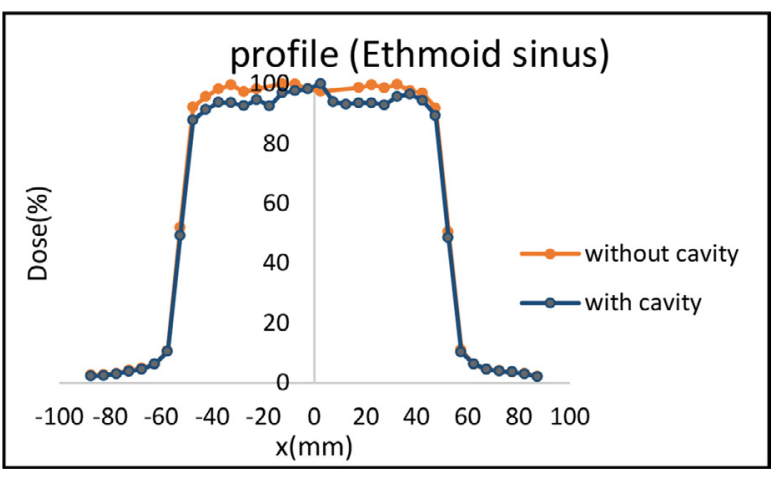

Figure 8: Comparing profile curves in the presence and absence of ethmoid sinus. 
calculations have been $\pm 1.75 \%$ and $\pm 1.6 \%$, respectively.

\section{Sphenoid sinus}

Figure 9 shows PDD values in the presence and absence of sphenoid sinus. When this air cavity is present, the maximum spot of the dose shifts from $1.4 \mathrm{~cm}$ to $1.5 \mathrm{~cm}$. We also see a $2.76 \%$ dose reduction before sphenoid sinus, a $13.01 \%$ dose reduction in air area, and a $2.25 \%$ increase in dose after air cavity.

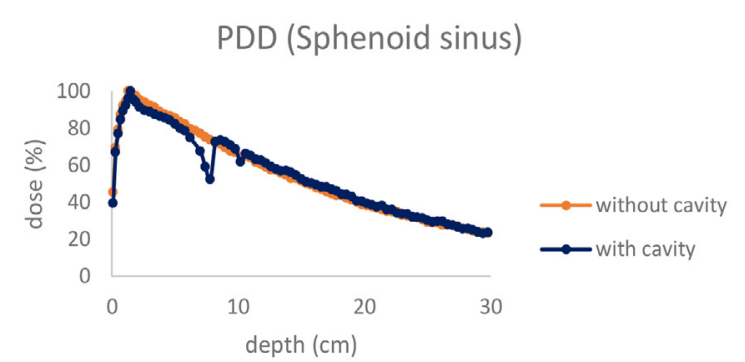

Figure 9: Comparing PDD curves in the presence and absence of sphenoid sinus.

Figure 10 demonstrates a comparison between dose profile values $(d=5 \mathrm{~cm})$ in the presence and absence of sphenoid sinus. As is seen in the Figure, since this sinus is located at depth of 6 to $8 \mathrm{~cm}$, significant changes cannot be seen in the profile.

The margins of error for PDD calculations and profile were $\pm 1.83 \%$ and $\pm 1.5 \%$, respectively.

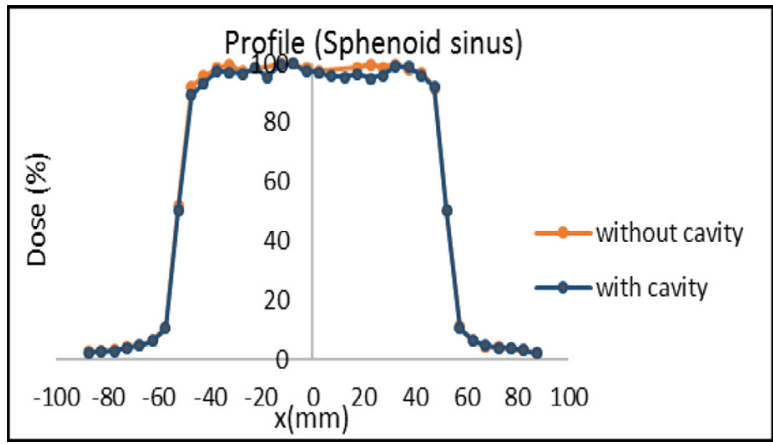

Figure 10: Comparing profile curves in the presence and absence of sphenoid sinus.

\section{Discussion and conclusion}

The present study examined the impact of air cavities of sinuses on radiotherapy dose distribution. The results indicate that the presence of air cavities leads to the generation of overdose and underdose regions in the intersection of air-tissue and it also is a contributing factor in inhomogeneity and fluctuation in dose distribution. The larger the cavity size is the more discontinuity and also fluctuations in the distribution of dose can be seen. Changes in dose distribution and dose fluctuation in the air cavity edges are quite evident.

Hideharu Miura et al. examined dose distribution in the presence of air cavities suggesting that dose reduction occurs right before the air cavity [2], which is in line with the present study. Results of this study indicate an increase in the maximum spot of the dose in the presence of cavity, which is in line with the results reported by Behrens. They investigated the dose build up in the presence of air cavities concluding that the dose build up increases in the presence of a very large air area with dimensions of $3 \times 16 \times 3 \mathrm{~cm}^{3}$ [13].

The results showed dose reduction before the air cavity and in the air area, which is dependent on geometry, size, and depth of the cavity. This decrease is due to the lack of scattered and returning beams. If the cavity dimensions get larger, the dose reduction before the air cavity and in the air area will be higher.

Also, the results indicated the dose increase after sinus cavities, which is dependent on geometry, size, and depth of the cavity. Since air cavities have a lower density than tissue, beam attenuation is lower in them; more beams pass through them and they also deposit the dose in a farther area. Therefore, the increase in absorbed dose takes place after the cavity. If the cavity gets larger, the absorbed dose increase will be higher. In other words, electronic balance is eliminated with the presence of these cavities near the intersection of air-tissue. Thus, the existence of sinus cavities, especially maxillary sinus, in the treatment field 
can disrupt therapeutic dose distribution, in a way that the impact of the cavity on therapeutic MU should be considered. When using the single-beam technique, adverse effects of air cavities on dose are more frequently observed. Using multiple-field techniques in designing a treatment planning, so that presence of the cavity with large dimensions in the treatment filed is avoided as much as possible, can lead to reductions in dose fluctuation.

\section{Acknowledgment}

This work was supported by Arak University of Medical Sciences [Grant number: 1920]. We deeply appreciate and thank the Department of Nuclear Engineering of the University of Science and New technologies of Esfahan For their co-operation in using their supercomputer system for the calculation of this research.

\section{Conflict of Interest}

None

\section{References}

1. Joshi CP, Darko J, Vidyasagar P, Schreiner LJ. Dosimetry of interface region near closed air cavities for Co-60, 6 MV and 15 MV photon beams using Monte Carlo simulations. Journal of Medical Physics/Association of Medical Physicists of India. 2010;35:73.doi: 10.4103/0971-6203.62197.

2. Miura H, Masai N, Yamada K, Sasaki J, Oh R-J, Shiomi $\mathrm{H}$, et al. Evaluation and commissioning of commercial Monte Carlo dose algorithm for air cavity. International Journal of Medical Physics, Clinical Engineering and Radiation Oncology. 2014;3:9.doi: 10.4236/ijmpcero.2014.31002.

3. Osei EK, Darko J, Mosseri A, Jezioranski J. EGSNRC Monte Carlo study of the effect of photon energy and field margin in phantoms simulating small lung lesions. Med Phys. 2003;30:2706-14. doi: 10.1118/1.1607551. PubMed PMID: 14596309.

4. Chow JC, Grigorov GN. Dosimetry of a small air cavity for clinical electron beams: A Monte Carlo study. Med Dosim. 2010;35:92-100. doi: 10.1016/j. meddos.2009.03.004. PubMed PMID: 19931020.

5. Li XA, Yu C, Holmes T. A systematic evaluation of air cavity dose perturbation in megavoltage x-ray beams. Med Phys. 2000;27:1011-7. doi: 10.1118/1.598966. PubMed PMID: 10841404.
6. Niroomand-Rad A, Harter KW, Thobejane S, Bertrand K. Air cavity effects on the radiation dose to the larynx using Co-60, $6 \mathrm{MV}$, and $10 \mathrm{MV}$ photon beams. International Journal of Radiation Oncology* Biology* Physics. 1994;29:1139-46.doi: 10.1016/0360-3016(94)90411-1.

7. Paelinck L, Reynaert N, Thierens H, De Wagter C, De Neve W. The value of radiochromic film dosimetry around air cavities: experimental results and Monte Carlo simulations. Phys Med Biol. 2003;48:1895905.doi: 10.1088/0031-9155/48/13/303.PubMed PMID: 12884923.

8. Shortt K, Ross C, Bielajew A, Rogers D. Electron beam dose distributions near standard inhomogeneities. Phys Med Biol. 1986;31:235.doi: 10.1088/0031-9155/31/3/003.

9. Zarza-Moreno M, Carreira $P$, Madureira L, Miras Del Rio H, Salguero FJ, Leal A, et al. Dosimetric effect by shallow air cavities in high energy electron beams. Phys Med. 2014;30:234-41. doi: 10.1016/j. ejmp.2013.07.125. PubMed PMID: 23920079.

10. Shahbazi $D$, Changizi $B$, Jomehzadeh $A$. The effect of contrast media on treatment planning and dose calculation in radiation therapy of pelvis cancers. $J$ Med Isfahan School. 2017;34:1389-94.

11. Epp ER, Lougheed MN, Mc KJ. Ionization build-up in upper respiratory air passages during teletherapy with cobalt 60 radiation. Br J Radiol. 1958;31:3617. doi: 10.1259/0007-1285-31-367-361. PubMed PMID: 13560735.

12. Petoukhova AL, Terhaard $\mathrm{CH}$, Welleweerd H. Does $4 \mathrm{MV}$ perform better compared to $6 \mathrm{MV}$ in the presence of air cavities in the head and neck region? Radiother Oncol. 2006;79:203-7. doi: 10.1016/j. radonc.2006.04.002. PubMed PMID: 16698100.

13. ehrens $C$. Dose build-up behind air cavities for Co-60, 4, 6 and 8 MV. Measurements and Monte Carlo simulations. Phys Med Biol. 2006;51:5937. doi: 10.1088/0031-9155/51/22/015.

14. Beach J, Mendiondo M, Mendiondo O. A comparison of air-cavity inhomogeneity effects for cobalt-60, 6-, and 10-MV x-ray beams. Med Phys. 1987;14:140-4.doi: 10.1118/1.596101.

15. Kan WK, Wu PM, Leung HT, Lo TC, Chung CW, Kwong DL, et al. The effect of the nasopharyngeal air cavity on x-ray interface doses. Phys Med Biol. 1998;43:529-37. PubMed PMID: 9533132.

16. Khan FM. The Physics of Radiation Therapy. Philadelphia; Lippincott, Williams \& Wilkins. 2010.

17. Klein EE, Chin LM, Rice RK, Mijnheer BJ. The influence of air cavities on interface doses for photon beams. Int J Radiat Oncol Biol Phys. 1993;27:41927.doi: $\quad 10.1016 / 0360-3016(93) 90255-x . P u b M e d$ PMID: 8407418. 